\title{
Primary Malignant Giant Cell Tumor of Bone: A Case Report
}

\author{
Guojun Zhou', Sen Li', Zhibin Li'²*, Qingpeng Deng², Peng Wang², Xiaowen Sang² \\ ${ }^{1}$ The First Clinical Medical College of Shaanxi University of Traditional Chinese Medicine, Xianyang 712046, Shaanxi \\ Province, China \\ ${ }^{2}$ Joint Ward, Orthopedics Hospital, Affiliated Hospital of Shaanxi University of Traditional Chinese Medicine, Xianyang \\ 712000, Shaanxi Province, China
}

*Corresponding author: Zhibin Li, 510505270@qq.com

\begin{abstract}
Primary malignant giant cell tumor of bone is clinically rare, lack of specificity, and often misdiagnosed. Currently, related literature about this tumor remains scarce. One case of primary malignant giant cell tumor of bone was diagnosed and treated in our hospital, and the treatment effect was satisfactory. There was no recurrence or metastasis in 2 years of followup. The report is as follows.
\end{abstract}

Keywords: Giant cell tumor of bone; Primary; Malignant; Distal femur; Tumor resection; Hinge knee replacement

Publication date: July 2021; Online publication: July 30, 2021

\section{Case data}

The subject of the present case report is a female, 55-year-old retired teacher, who was admitted to the hospital in January 2019 with the complaint of "left knee soreness for 20 years, aggravated with limp for 2 months." Physical examination revealed claudication while walking, tenderness on left knee, percussion pain, medial condyle palpable mass, and strongly positive result from patella grinding test. Digital radiography (DR) showed tumorous lesions in the lower part of the left femur, with a possibility of a giant cell tumor of bone; malignancy is not ruled out (Figure 1A-B). Computed tomography (CT) scan showed the condyle-like lesions of the left femur (Figure 1C). Magnetic resonance imaging (MRI) showed bone space-occupying lesions in the lower segment of the left femur (Figure 1D-G). Single photon emission tomography/computed tomography (SPET/CT) showed tumorous lesions of the left distal femur bone (Figure 1H). After a comprehensive analysis after admission, surgical contraindications were excluded. A needle biopsy revealed malignant giant cell tumor of bone, and the tumor series were normal. Our diagnosis of this case was primary malignant giant cell tumor of bone in the distal left femur (G2T2M0 stage).

In January 2019, the patient underwent general anesthesia with tumor resection and hinge knee replacement. After successful general anesthesia, with the use of routine sterilized drapes, anterior median incision was performed to fully expose the distal femur and proximal tibia, and the proximal femur $5 \mathrm{~cm}$ away from the tumor which was to be resected. The tumor tissue is shown in Figure 1I. Appropriate hinged knees were used as femoral prosthesis, tibial prosthesis was installed after tibial plateau osteotomy, spacers were placed, and the knee joint was moved without abnormality. The prosthesis was in good position under fluoroscopy, and the operation was smooth with routine irrigation and suture.

Postoperative pathological results showed malignant giant cell tumor of bone in the left distal femur with extensive hemorrhagic cystic degeneration (Figure 1J). Immunohistochemical results are as follows: H3.3 G34W (+), H3.3 G34V (-), P63 (scattered +), SATB2 (-), and SOX9 (-). After the operation, the 
patient was given treatments, such as infection prevention, fluid rehydration, and analgesia, and she was discharged from the hospital after recovery. The oncology department received regular chemotherapy, supplemented by local radiotherapy. After more than 2 years of outpatient follow-up, there was no recurrence or metastasis of the tumor. Repeated examinations of DR showed that the left knee was changed after the knee arthroplasty and the position was good (Figure 1K-L).
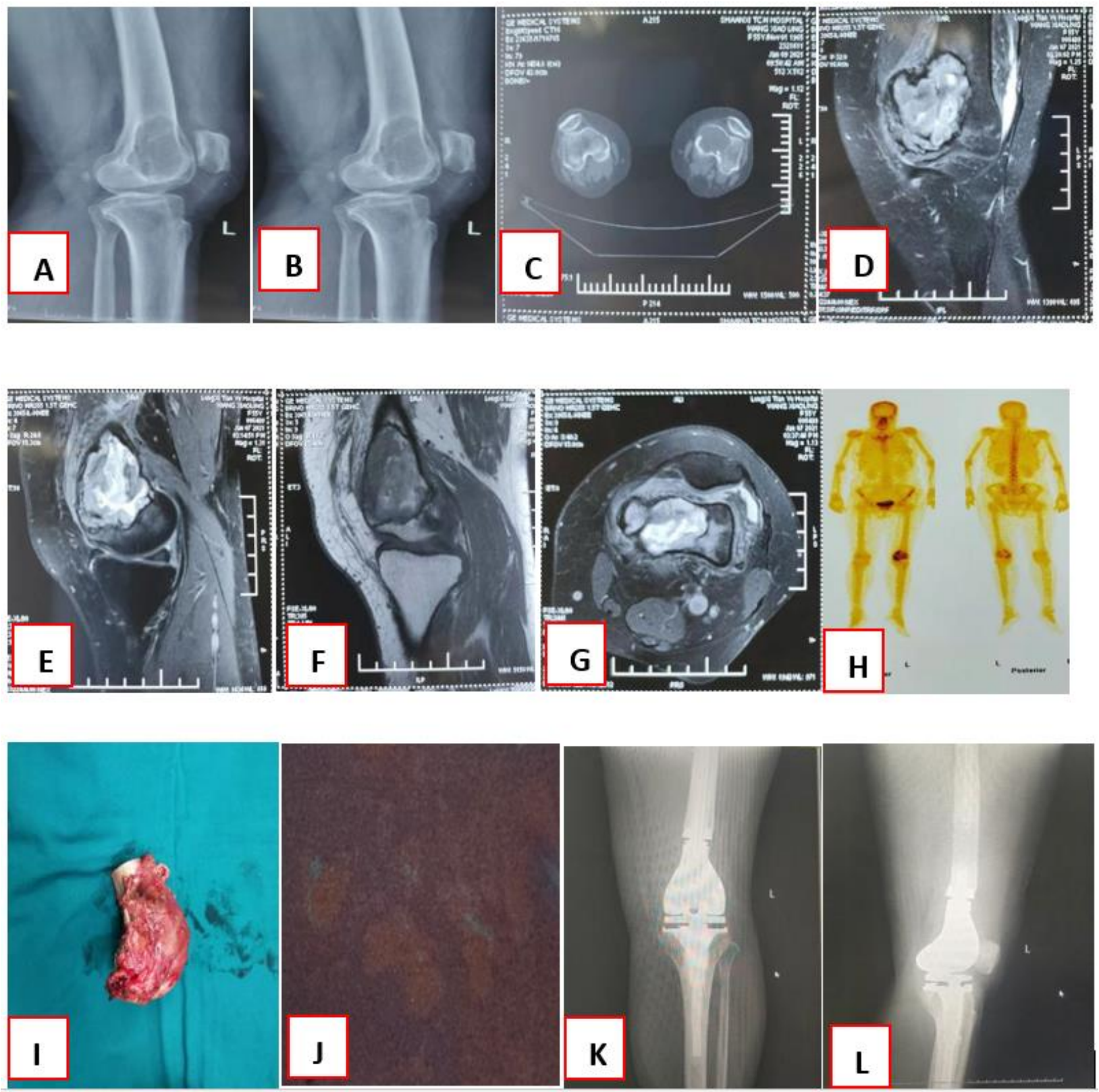

Figure 1. (A,B) Tumorous lesions of the left lower femur, giant cell tumor of bone, and probable malignancy. (C) Left femoral condyle tumor-like lesions are likely. (D-G) Left lower femur bone space-occupying lesions. (H) Tumorous lesions of bone in the distal left femur. (I) Tumor body tissue. (J) Malignant giant cell tumor of bone with extensive hemorrhagic cystic degeneration. (K-L) The left knee has been changed after arthroplasty and the position is good.

\section{Case discussion}

Malignant giant cell tumors of bone (MGCTB) is a malignant osteolytic tumor that originates from cancerous bone. It is highly aggressive and recurring. It is named so because of its large cell size. MGCTB 
is clinically rare and lacks specificity ${ }^{[1]}$, accounting for about $1 \%$ to $5 \%$ of the total number of giant cell tumors of bone ${ }^{[2]}$. The clinical symptoms of MGCTB are not obvious in the early stage, but become more common in the late stage with severe pain, swelling and lumps. There is currently standard diagnosis and treatment system for MGCTB yet, and doctors mostly rely on pathological diagnosis and surgical treatment.

The pathogenesis of MGCTB is currently unclear, and the diagnosis mainly depends on pathological diagnosis and immunohistochemistry. MGCT and benign GCTB are difficult to distinguish in clinical manifestations and imaging. If the following signs appear, malignancy is highly suspected: (i) The bone crest is arranged disorderly and has an irregular shape ${ }^{[3]}$; (ii) There is infiltrating bone destruction, the boundary of the bone shell is unclear, and the boundary seems worm-eaten and sieve-shaped bone destruction area; (iii) The soft tissue mass exceeds the mutilated bone shell; (iv) The bone cortex is destroyed, exhibiting small patchy periosteal reaction; (v) Intolerable severe pain. MGCTB is characterized by the presence of multinucleated giant cells and aggressive clinical course from histological analysis. Zang et al. ${ }^{[3]}$ analyzed 12 cases of MGCTB confirmed by surgery and pathology and found that under the light microscope of MGCTB, the number of mononuclear stromal cells was significantly increased, the arrangement was disordered, and mitoses were more common; the number of giant cells was small and the size was small. The patient's intraoperative measurement of the tumor tissue was about $6.3 \mathrm{~cm} \times 5.1 \mathrm{~cm} \times$ $5.6 \mathrm{~cm}$, accompanied by swelling and infiltrating growth, soft and fragile center of the tumor, extensive bleeding and cystic change. The tumor showed irregular shape, bulging and polycystic change. Bone destruction is obvious, and there are no metastatic lesions throughout the body, which may be consistent with the early manifestations of MGCTB. Pathological diagnosis is the gold standard for diagnosis of this disease. According to the Enneking staging of bone tumors, it is G2T2M0 stage.

There is currently no diagnosis and treatment guideline for MGCTB. The preferred treatment is to adopt expanded resection, but limb function should be preserved as much as possible. Jing et al. ${ }^{[4]}$ used customized rotary hinge knee prosthesis to treat 14 cases of knee malignant bone tumors, and the 3-year follow-up survival rate was $92.9 \%$. After analyzing 28 cases of proximal fibula bone tumors, He et al. ${ }^{[5]}$ concluded that the larger the tumor mass, the higher the malignancy, and the less sensitive to chemotherapy, the more suitable for tumor patients to undergo extended resection. In this case, the puncture examination revealed malignant osteocytoma; combined with clinical symptoms, imaging features, left knee joint degeneration, etc., it is appropriate to adopt the resection of the tumor segment and the rotating hinge artificial knee replacement. This operation not only has the advantages of restoring joint function, eliminating joint pain, and correcting limbs, but also has the advantages of thorough tumor resection and low recurrence rate. It also guarantees the quality of life of patients and solves the problem of knee osteoarthritis in elderly patients. Curettage and bone grafting or extensive resection of the lesion is not indicated for MGCTB as it is prone to recurrence and metastasis after surgery. If the patient is young, it will do more harm than good after amputation.

In summary, MGCTB is a clinically rare malignant osteolytic tumor that is prone to recurrence and metastasis. Pathological means is the gold standard for the diagnosis of this disease. At present, radical surgical treatment is mostly used, supplemented by radiotherapy and chemotherapy when necessary. After comprehensive analysis of this case, the tumor segment resection and rotary hinge artificial knee replacement surgery was adopted, and the therapeutic effect was satisfactory.

\section{Funding}

A clinical study on the treatment of knee osteoarthritis by Kangjian's three-dimensional model (2020XZ003) 


\section{Disclosure statement}

The authors declare that there is no conflict of interest.

\section{References}

[1] Wu WB, Peng T, et al., 2017, Imaging Characteristics and Misdiagnosis Analysis of Giant Cell Tumor of Bone. CT Theory and Application Research, 26(4): 505-510.

[2] Bertoni F, Bacchini P, Staals EL, 2003, Malignancy in Giant Cell Tumor of Bone. Cancer, 97(10): 2520-2529.

[3] Zang YW, Lou HA, 2021, Imaging Manifestations of Malignant Giant Cell Tumor of Bone. Journal of Qingdao University (Medical Edition), 1-6.

[4] Jing L, Zhang HM, Han L, et al., 2015, Total Knee Arthroplasty for Knee Joint Malignant Bone Tumors. Chinese Journal of Bone and Joint Surgery, 8(05): 425-428.

[5] He ZW, Fan GT, Zhang JL, et al., 2020, Clinical Analysis of Surgical Resection for Malignant Tumors of The Proximal Fibula. Chinese Journal of Bone and Joint, 9(05): 336-340. 